\title{
Establishment of an animal model of spontaneous cervical lymph node metastasis of laryngeal squamous cell carcinoma and obtaining laryngocarcinoma cells with high metastatic potential
}

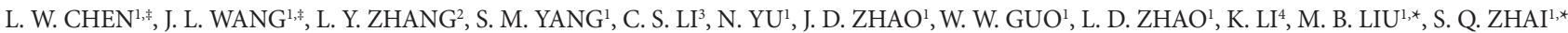

${ }^{1}$ Department of Otolaryngology Head and Neck Surgery, Chinese PLA General Hospital, Beijing 100853, P. R. China; ${ }^{2}$ Department of Pharmacology and Chemical Biology, University of Pittsburgh, E1301 BSTWR 200 Lothrop Street, Pittsburgh, PA 15260, USA; ${ }^{3}$ Department of Respiratory Diseases, Chinese PLA General Hospital, Beijing 100853, P. R. China; ${ }^{4}$ Department of Neurological Surgery, Weifang Medical College, Weifang, Shandong 261053, P. R. China

*Correspondence: mingboliu@yahoo.com.cn, zhaisq@plagh.com.cn

${ }^{*}$ Contributed equally to this work.

Received December 9, 2012/ Accepted March 8, 2013

\begin{abstract}
To establish an animal model of spontaneous cervical lymph node metastasis of laryngeal squamous cell carcinoma and obtain laryngocarcinoma cells with high metastatic potential, laryngeal squamous cell carcinoma cell line HEP-2 in logarithmic phase were inoculated under the lingual margin mucosa of nude mice. HEP-2 cells metastasized to the cervical lymph nodes were isolated, cultured, and re-inoculated under the lingual margin mucosa of nude mice twice. The tumor formation in the tongue and in the cervical lymph nodes was confirmed by pathological examination. Carcinoma cells' ability of invasion and migration was detected by transwell assay. Human specific Alu sequences were detected by PCR, which indicated that the tumor cells originated from human laryngeal squamous cell carcinoma cell line HEP-2. Finally, an animal model of spontaneous lymph node metastasis of laryngeal squamous cell carcinoma was successfully established. Laryngeal squamous cell carcinoma cells with high metastatic potential to lymph nodes were obtained through repeated inoculations.
\end{abstract}

Key words: laryngeal cancer, lymph node metastasis, animal model

Laryngocarcinoma is a common malignant neoplasm of the head and neck. The most commonly encountered laryngocarcinoma is laryngeal squamous cell carcinoma, which accounts for more than $95 \%$ laryngocarcinomas, and the incidence of this disease has been increasing in recent years. The survival rate of laryngocarcinoma patients has not been improved significantly, although surgical and radiochemotherapy techniques have improved remarkably. According to the statistics, the total survival rate of patients with various organ malignant neoplasms has improved in the US in recent years. However, the survival rate of laryngocarcinoma patients did not show any increase, and rather was decreased [1,2].

Local invasion and cervical lymph node metastasis occur easily in laryngocarcinoma patients. The common cause of mortality in laryngocarcinoma patients is tumor recurrence in the neck, and metastasis. Two main pathways for invasion and metastasis of tumor cells are lymph and blood vessels, which mediate distant metastasis of most solid tumors. Many studies have shown that the metastasis of most epithelial origin tumors occurs through lymphatic vessels at an early stage, and the metastasis of more than $50 \%$ tumors is through lymphatic vessels. Most metastasis of laryngocarcinoma occurs in the cervical lymph nodes. One important basis for selecting the treatment for laryngocarcinoma is whether or not cervical lymph node metastasis is present.

In order to analyze the molecular and cellular mechanisms involved in laryngocarcinoma metastasis, we established an animal model of spontaneous cervical lymph node metastasis of laryngeal squamous cell carcinoma and screened for the carcinoma cells with high metastatic potential. This provided a novel technical way to investigate the molecular pathways involved in the growth and metastasis of laryngeal squamous cell carcinoma in vivo and in vitro. 


\section{Materials and methods}

Experimental animals. 4-week-old $\mathrm{Bal} / \mathrm{Bc}$ nude mice, with a body weight of 12-14g of either sex were supplied by Institute of Zoology, Academy of Military Medical Sciences, China. The animals were fed within an ultraclean specific pathogen free (SPF) laminar flow rack under constant temperature $\left(20-26^{\circ} \mathrm{C}\right)$ and constant humidity (40\%-50\%) conditions. The animals had free access to sterilized water and feed.

Cell culture. Human laryngeal squamous cell carcinoma cell line HEP-2 was provided by Institute of Basic Medical Science, Chinese Academy of Medical Sciences and Peking Union Medical College. HEP-2 cells were routinely grown in DMEM medium (100 U of penicillin and $100 \mu \mathrm{g} / \mathrm{ml}$ of streptomycin) with $10 \%$ fetal bovine serum (Gibco-BRL, Grand Island, NY, USA) at $37^{\circ} \mathrm{C}$ in a constant temperature incubator containing 5\% $\mathrm{CO}_{2}$. The cells in logarithmic phase were digested with tripsin and collected. The density of collected cells was adjusted to $1.5 \mathrm{x} 10^{8} / \mathrm{ml}$ with sterilized normal saline for subsequent experiments. The viable cell count was over $95 \%$ as determined by trypan blue staining.

Tumor cell inoculation. The nude mice were anesthetized via intraperitoneal injection of $1.25 \%$ napental $(35 \mathrm{mg} / \mathrm{kg}$ body weight). After successful anesthesia, $10 \mu \mathrm{l}$ of the cell suspension as described above was injected under the lingual margin mucosa of each nude mouse using a $50 \mu \mathrm{l}$ microinjector. Twenty mice were inoculated each time. Two nude mice were randomly selected as controls and they did not undergo any treatment. The growing status of these nude mice was carefully monitored.

Pathological assay. When the tumor was formed in the tongue of the nude mice, and the mice showed emaciation and were in a dying state, they were sacrificed. The neoplasm in the tongue and the swollen lymph nodes were fixed in $4 \%$ paraformaldehyde, and then the fixed tissue was dehydrated, cleared, and embedded in paraffin. The paraffin-embedded tissue blocks were successively cut into sections, which were then baked in a slide drier at $60^{\circ} \mathrm{C}$ for $4 \mathrm{~h}$. After conventional hematoxylin and eosin ( $\mathrm{HE}$ ) staining, the status of the tumor formation in the tongue and the cervical lymph nodes were observed under a light microscope.

Culture of tumor cells metastasized to lymph node. When the mice showed emaciation and were in a dying state, they were sacrificed and the cervical lymph nodes were excised in a superclean bench and cut into pieces on a 150-mesh sieve. The cells were collected with PBS and centrifuged, and then re-suspended in DMEM medium (100 U of penicillin and $100 \mu \mathrm{g} / \mathrm{ml}$ of streptomycin) with $10 \%$ fetal bovine serum. The cells were cultured at $37^{\circ} \mathrm{C}$ in an incubator containing $5 \% \mathrm{CO}_{2}$ for about 10 days. When the cell confluence reached $80 \%$, cells were passaged for further culture. After repeated passages, stably growing tumor cells were obtained.

Extraction of DNA from cells and amplification of human Alu sequences with PCR. The DNA from cells was extracted using a genomic DNA extraction kit (centrifugecolumn type, Tiangen, China). For amplifying the 221-bp sequence for human specific Alu, the primers were as follows: forward, 5'-CACCTGTAATCC CAGCAGTTT-3'; reverse, 5'CGCGATCTCGGCTCACTGCA-3'. The amplification cycle conditions were as follows: denaturing at $95^{\circ} \mathrm{C}$ for $5 \mathrm{~min}$, then $94^{\circ} \mathrm{C}$ for $1 \mathrm{~min}, 57^{\circ} \mathrm{C}$ for $1 \mathrm{~min}, 72^{\circ} \mathrm{C}$ for $1 \mathrm{~min}$ for a total of 30 cycles, then extension at $72^{\circ} \mathrm{C}$ for $5 \mathrm{~min}$. Next, $5 \mu \mathrm{l}$ of the PCR amplification products underwent $1.5 \%$ agarose gel electrophoresis for 20-30 min. The amplified human Alu sequences were observed and identified with a UV spectrophotometer.

Cell migration and invasion assay. Matrigel $(1 \mathrm{mg} / \mathrm{mL}$; BD Biosciences, Bedford, MA) was used to cover the chambers of the Transwell inserts (Corning, Corning, NY), which were then put into a 24 -well plate. HEP-2 cells $\left(10^{5} / 100 \mu \mathrm{l}\right)$ in DMEM were added to the upper chamber and DMEM containing $10 \%$ FBS was added to the lower chamber, followed by culture for $48 \mathrm{~h}$. Then, the cells were fixed in $75 \%$ methanol followed by staining with $0.1 \%$ crystal violet. The procedures for migration detection were similar to those of invasion detection except that the chambers were not coated with Matrigel. In addition, $5 \times 10^{4} \mathrm{HEP}-2$ cells were seeded followed by incubation for $12 \mathrm{~h}$ and subsequent cell counting.

\section{Results}

Animal model of spontaneous cervical lymph node metastasis of laryngeal squamous cell carcinoma. Approximately 4 weeks after injection of tumors cells under the lingual margin mucosa of the nude mice, tumors were formed obviously in the tongue of the mice. The tumor formation rate was 100\% after injection. The nude mice had difficulty eating, became emaciated, and went into a dying state. After the mice were sacrificed, their necks were dissected. One or several swollen lymph nodes could be found in the necks of $70 \%$ of the inoculated nude mice. The swollen lymph nodes were removed, and the tumor cells metastasized to the lymph nodes were isolated and cultured. The isolated cells were re-inoculated, and cervical lymph node metastasis occurred in $85 \%$ of re-inoculated nude mice. Carcinoma cells acquired by the previous procedure were inoculated for the third time. Tumor cells metastasized to the lymph nodes were isolated and cultured again. This time, ratio of cervical lymph node metastasis was $95 \%$. The carcinoma cells that easily metastasized to the lymph nodes were obtained finally. The tumor formation and the ratio of lymph node metastasis in three inoculations were shown in Fig. 1 and Tab 1.

Table 1. Ratios of spontaneous cervical lymph node metastasis of laryngeal squamous cell carcinoma.

\begin{tabular}{lccc}
\hline Cell lines & $\begin{array}{c}\text { No. of } \\
\text { total mice }\end{array}$ & $\begin{array}{c}\text { No. of mice with cervical } \\
\text { lymph node metastasis }\end{array}$ & Ratio \\
\hline $\begin{array}{l}\text { Hep-2 } \\
\begin{array}{l}\text { Tumor cells metastasized to } \\
\text { the cervical lymph nodes }\end{array}\end{array}$ & 20 & 14 & $70 \%$ \\
$\begin{array}{l}\text { Tumor cells metastasized to } \\
\text { the cervical lymph nodes }\end{array}$ & 40 & 17 & $85 \%$ \\
\hline
\end{tabular}

* : Tumor cells metastasized to the cervical lymph nodes for the first time **: Tumor cells metastasized to the cervical lymph nodes for the second time 

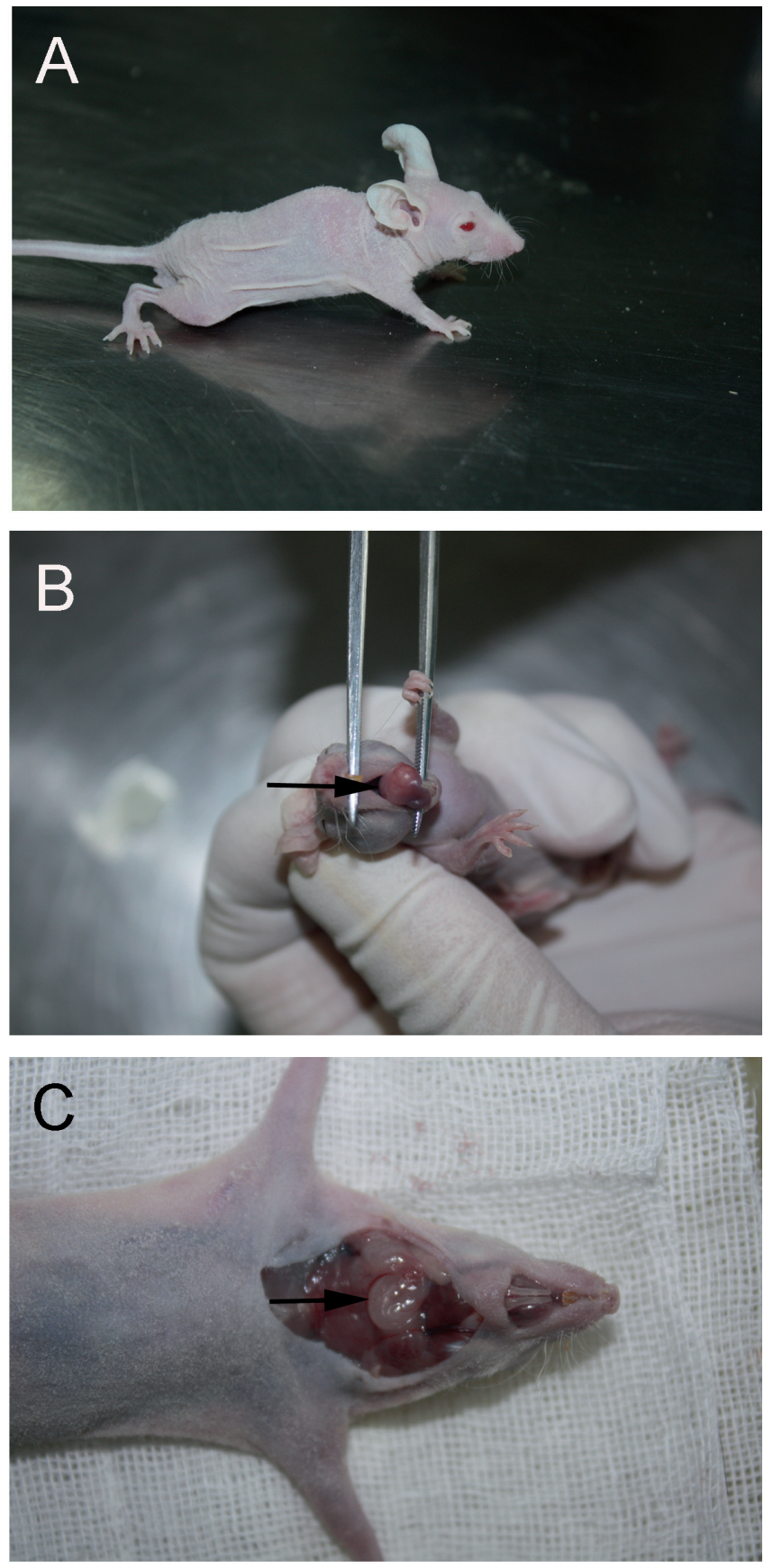

Figure 1. Illustration of tumor formation in the tongue and swollen lymph nodes in the neck of the nude mice. A: Emaciated nude mice which was on the verge of death after HEP-2 cells were injected in to the tongue; $B$ : The neoplasm in the tongue, $\rightarrow$ :neoplasm; C: The lymph nodes in the neck become swollen after tumor cells metastasized from the neoplasm in the tongue. $\rightarrow$ :swollen lymph nodes.

Pathological examination of tumor formation in the tongue and swollen lymph nodes in the neck of nude mice. The tongue neoplasms and swollen lymph nodes of the experimental groups and normal tongues and lymph nodes of the control group were collected, processed with HE staining, and then observed under light microscopy. Solid tumors with a spherical shape were formed under the lingual margin mucosa in nude mice of the experimental groups, and tumor cells with a larger volume, various shapes, and abnormal nuclear division were diffusely arranged. After staining the swollen lymph nodes, diffuse infiltration of tumor cells and disorganization of the normal internal structure of the lymph nodes was observed (Fig. 2).

Identification of the obtained carcinoma cells' ability of migration and invasion. Cell migration and invasion were determined by transwell assays. We found that the ability of migration and invasion of the obtained carcinoma cells by this animal model were significantly stronger than the parental tumor cells of HEP-2 (Fig. 3).

Obtaining and identification of laryngeal squamous cell carcinoma cells with high metastatic potential. The laryngeal squamous cell carcinoma cells metastasized to the cervical lymph nodes were isolated and cultured under aseptic conditions, and then observed under light microscopy. Compared with parental HEP-2 cells, these tumor cells had a slightly larger volume with a roughly round shape. The genome DNA was extracted from the final tumor cells obtained, and the 221-bp specific human Alu sequence was amplified by PCR (Fig. 4), confirming that the isolated and cultured tumor cells metastasized to the cervical lymph nodes were originally from the human cell line HEP-2.

\section{Discussion}

For clinical oncologists, treating tumor metastasis is one of the most difficult tasks. Patients with distant metastasis lose the opportunity of surgical treatment, and about $90 \%$ patients with malignancies die of tumor metastasis [3]. Local metastasis is the main cause of tumor recurrence and death, and cervical lymph node metastasis suggests a poor prognosis. Although metastasis is the main cause of death in solid tumor patients, the molecular and cellular mechanisms involved in tumor metastasis are not yet understood $[4,5,6]$.

Many researchers investigated the mechanisms of tumor invasion and metastasis, and explored new means of tumor treatment by establishing animal models. The current animal models for tumor research can be mainly classified into three categories: spontaneous tumor model, induced tumor model, and implanted tumor model, which includes homograft and xenograft tumors. An individual model can be selected according to the requirements of different experiments. Nude mice are the most popular experimental animals in tumor research since they have innate immunodeficiency, and tumor cells can be successfully implanted. Nude mice are an excellent tool for studying tumor development and progression. Povlsen and Rygaard [7] carried out the first oncological experiment to inoculate human tumor cells into nude mice. Braakhuis et al [8]investigated the biological characteristics of head and neck tumors through xenograft with cells from human head 

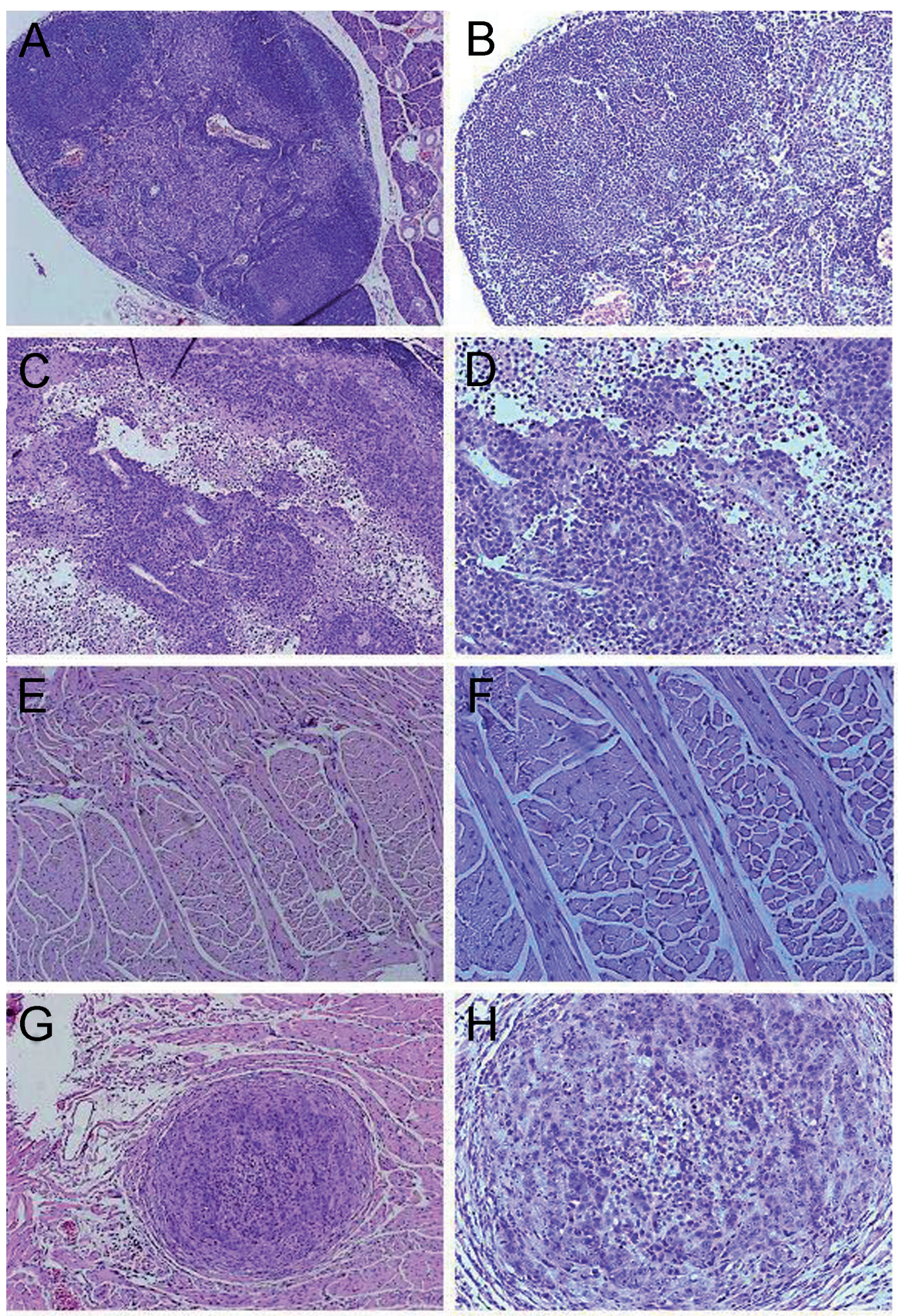

Figure 2. Pathological sections of the tongue and cervical lymph nodes(H\&E). A\&B: Normal lymph node in the neck of nude mice $(\times 100 ; \times 200)$; C\&D: Swollen lymph node in the neck after tumor cells metastasized from the tongue $(\times 100 ; \times 200)$; E\&F: Normal tongue of the nude mice $(\times 100$; $\times 200)$; G\&H: Neoplasm in the tongue $(\times 100 ; \times 200)$. 
A

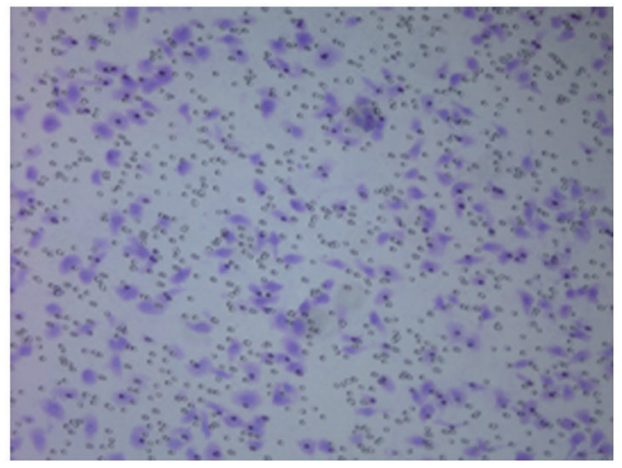

HEP-2

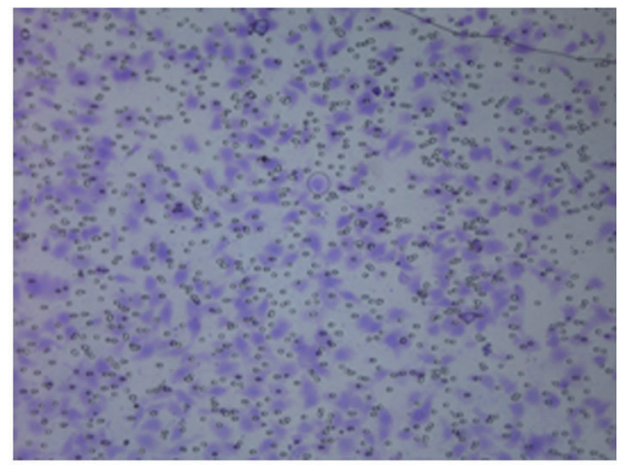

HEP-2 with high

metastasis ability

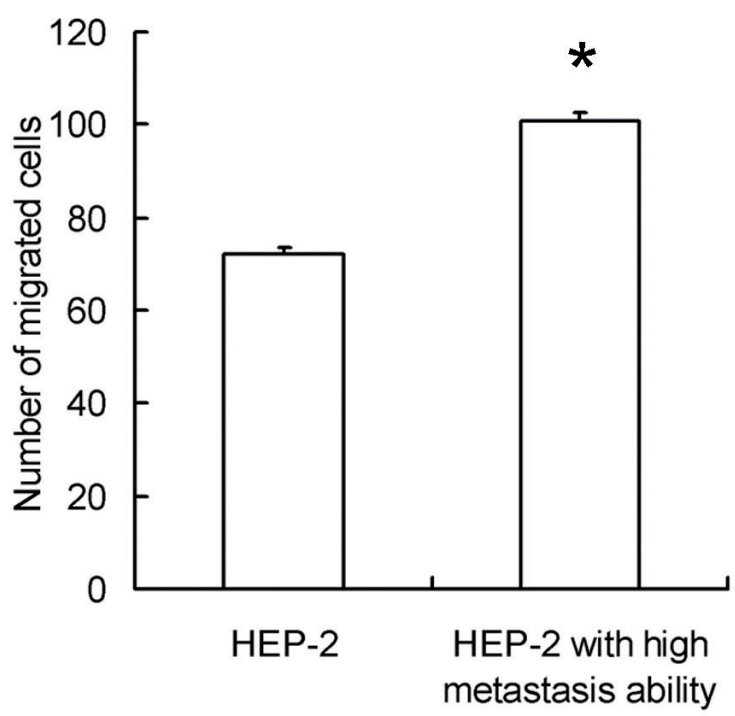

B

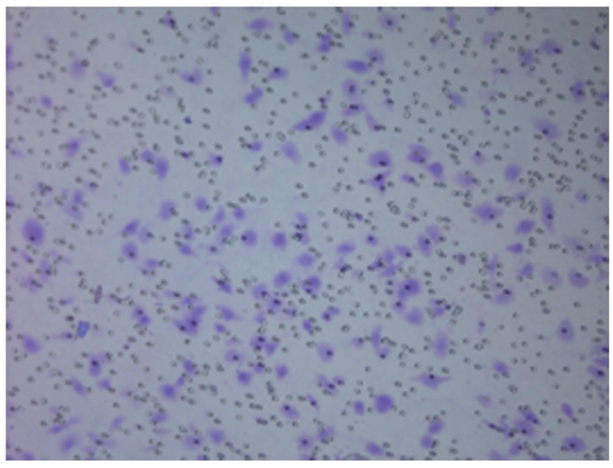

HEP-2

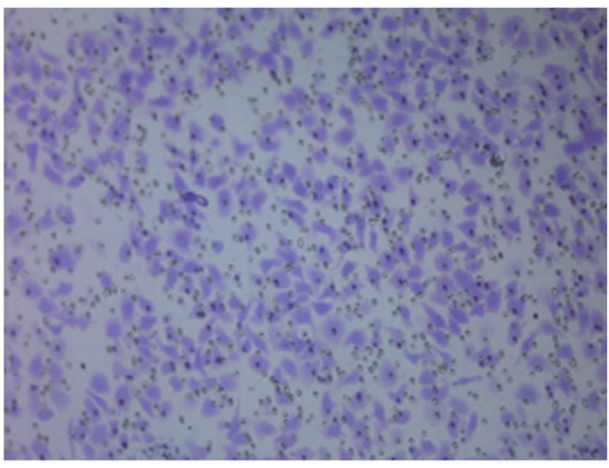

HEP-2 with high

metastasis ability

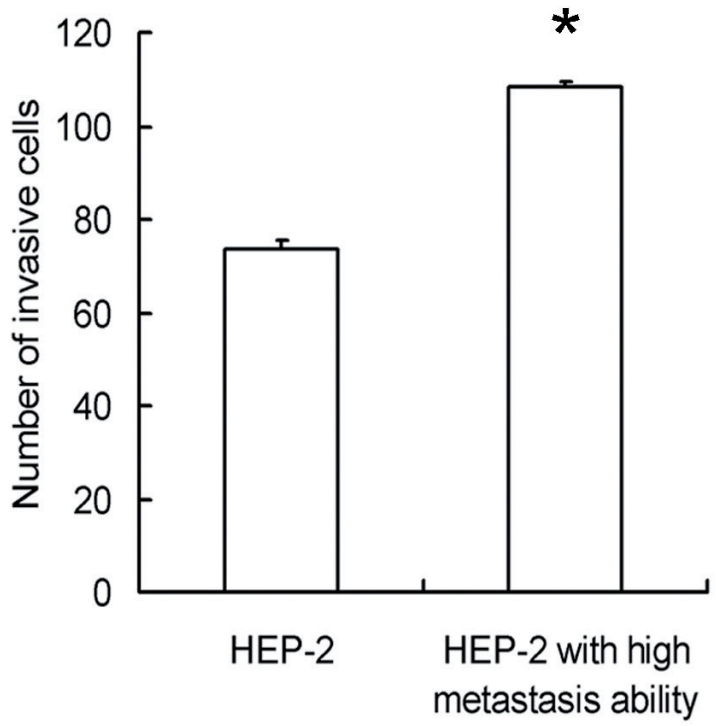

Figure 3. Cell invasion and migration ability detection by transwell assay. A: Cell migration of tumor cells metastasized to the cervical lymph nodes for the third time of inoculation were stranger than HEP-2 cells $(p<0.05)$. B: Cell invasion of tumor cells metastasized to the cervical lymph nodes for the third time of inoculation were much mor stranger than HEP-2 cells $(\mathrm{p}<0.05){ }^{\star}: \mathrm{P}<0.05$. 

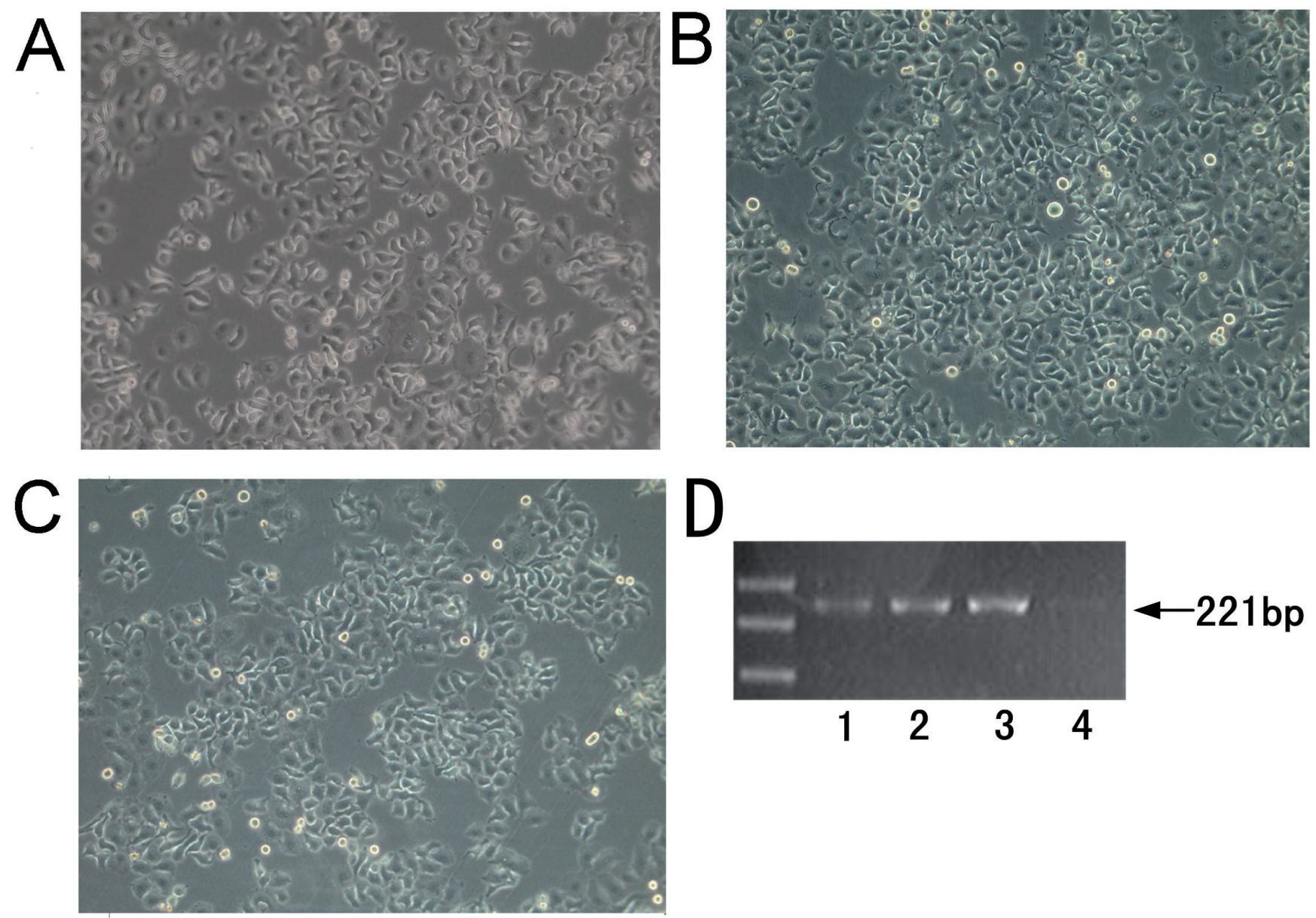

Figure 4. Laryngocarcinoma cells with high metastatic potential obtained from the nude mice model of lymph node metastasis. A: Tumor cells metastasized to the cervical lymph nodes for the first time of inoculation; B: Tumor cells metastasized to the cervical lymph nodes for the second time of inoculation; C: Tumor cells metastasized to the cervical lymph nodes for the third time of inoculation; D: PCR amplified 221bp products of human-specific Alu sequence of the cells isolated from the swollen lymph node. 1:Tumor cells metastasized to the cervical lymph nodes for the second time of inoculation; 2:Tumor cells metastasized to the cervical lymph nodes for the third time of inoculation;3.HEP-2 cells as positive control; 4.Negative control.

and neck tumors. The traditional transplantation process was subcutaneous transplantation of tumor cells or tissues. However, since the subcutaneously transplanted tumors could often be covered by fibrous connective tissue, local diffusion and spontaneous metastasis of tumors were limited. Therefore, it was not an ideal model for studies of the mechanisms of tumor metastasis. Later, some researchers reported that a model of tumor metastasis could be successfully established by inoculating tumor cells at the primary sites. Kawasashiri et al [9] reported that when oral tumor cells were inoculated under the tongue of nude mice, lymph node and lung metastasis occurred. However, since the squamous cell carcinoma used by Kawasashiri was highly malignant and multiple organ metastases easily occurred, some investigators considered that it could not represent the standard mode of oral cancer metastasis. Myers et al.[10] and Patell et al [11] successfully applied inoculating head and neck squamous cell carcinoma cells at the primary sites to study the role of head and neck squamous cell carcinoma respectively, they both found much more stable results than that of those inoculated under the skin.

The model of spontaneous lymph node metastasis of laryngocarcinoma established in this study was based on the concepts described above. Since laryngocarcinoma occurs at a key site in the respiratory tract, the biggest obstacle to orthotopic transplantation of tumors is that the tumor will lead to death due to suffocation before metastasis of the tumor occurs. Studies on the biological specificities of laryngocarcinoma can be carried out by inoculating laryngocarcinoma cells under the lingual mucosa of nude mice due to the similarity between the lingual mucosa and the laryngeal mucosa.

The animal model established in this study was a xenograft tumor model. There has been no report of a similar animal model in laryngocarcinoma research. A similar approach for investigating the biological characteristics of tumors has only been reported in studies of oral squamous cell carcinoma. Umeda et al [12] established a model of lymph node metastasis 
of oral cancer by inoculating tumor cells and tissues into the tongues of nude mice. The survival rate of tumor cells was $50 \%$ $83 \%$, and the rate of lymph node metastasis was only $13 \%-40 \%$. Sun et al [13] established animal models of cervical lymph node metastasis in nude mice by inoculating oral squamous cell carcinoma cells into the tongue, mouth floor, or axilla. The rate of lymph node metastasis of tumor cells inoculated into the mouth floor was highest (55.6\%), and the metastatic tumor cells showed typical biological atypia. In our studies, when 1 $\mathrm{x} 10^{6}$ cells were inoculated under the lingual margin mucosa, the rate of tumor formation in the tongue was $100 \%$, the rate of cervical lymph node metastasis of parent HEP-2 cells was $70 \%$, and rate of cervical lymph node metastasis of second collected tumor cells with high metastatic potential was $95 \%$. The experimental data demonstrated that tumor formation in this animal model was stable, and rate of cervical lymph node metastasis was high. Thus, this model could become a powerful tool for studying molecular biological mechanisms involved in lymph node metastasis of laryngocarcinoma.

In this study, we have successfully obtained squamous cell carcinoma cells metastasized to cervical lymph nodes, which were frozen in liquid nitrogen after repeated passages and subculture and could be used as a new research tool for subsequent studies on cellular biological characteristics of invasion and metastasis of laryngocarcinoma cells. Matsui et al [14] obtained tumor cells through inoculating oral squamous carcinoma cell line HSC-3 into the tongue of nude mice, followed by isolating metastatic tumor cells from the cervical lymph nodes after 3 weeks. Then, oral squamous cell carcinoma cells with high metastatic potential were obtained after three repeated inoculations. In our study, we repeated inoculation and screening three times too. Our main concern was that the malignant degree of tumor cells would be increased, and the biological characteristics of these cells would be altered if more passages and subcultures were performed. While we did our best to screen for the tumor cells with the highest metastatic potential to lymph node, we also wanted to maintain the oncological properties of the parent tumors to facilitate our subsequent analysis on the biological factors involved in tumor cell metastasis. After get the carcinoma cells, cell invasion and migration were detected by transwell assay. The obtained carcinoma cells show much more property of invasive and metastatic(both $\mathrm{P}<0.05)$. With immunohistochemical analysis and PCR, the tumors formed in the tongue and cervical lymph nodes demonstrated to have features of squamous cell carcinoma, and the tumor cells isolated from the cervical lymph nodes were confirmed to contain human specific Alu sequences and therefore originate from human tumor cells.

In conclusion, we established for the first time an animal model of spontaneous cervical lymph node metastasis of laryngeal squamous cell carcinoma, and obtained laryngeal squamous carcinoma cells with high metastatic potential to lymph nodes. Our results provide a novel method for studies on lymph node metastasis of laryngocarcinoma. The molecular mechanisms involved in the regulation of invasion and metastasis of laryngocarcinoma cells can be explored, and key regulators can be found by investigating the features of tumor cells with high metastatic potential to lymph nodes.

Acknowledgements: This project was supported by the Natural National Science Fundation of China (No. 30872853). We thank Mr. Yongquan Wang and Mrs. Zhikai Han for their technical assistance.

\section{References}

[1] REBECCA S, DEEPA N, AHMEDIN J. Cancer Statistics,2012. CA CANCER J CLIN 2012; 62: 10-29.

[2] OLSEN KD. Reexamining the treatment of advanced laryngeal cancer. Head \& Neck 2010; 32: 1-7.

[3] YILMAZ M, CHRISTOFORI G. EMT, the cytoskeleton, and cancer cell invasion. Cancer Metastasis Rev 2009; 28: 15-33.

[4] FIDLER I. J. The pathogenesis of cancer metastasis: the ,seed and soil' hypothesis revisited. Nat Rev Cancer 2003; 3: 453-458.

[5] WEIGELT B, PETERSE JL, VAN ,T VEER LJ. Breast cancer metastasis: markers and models. Nat Rev Cancer 2005; 5: 591-602.

[6] GUPTA, GP, MASSAGUE, J. Cancer metastasis: building a framework. . Cell 2006; 127: 679-695.

[7] Povlsen CO, Rygaard J: Heterotransplantation of a human malignant tumor to nude mice. Acta Pathol Microbial Scand 1972; 80: 713-717.

[8] BRAAKHUIS BJ, SNEEUWLOPER G, SNOW GB. The potential of the nude mouse xenograft model for the study of head and neck cancer. Arch Otorhinolaryngol 1984; 239: 69-79.

[9] KAWASHIRI S, KUMAGAI S, Ye F, KOJIMA S, YAMAMOTO E. Experimental studies on the mechanism of invasion and metastasis of oral squamous cell carcinoma. Part III: A new spontaneous metastasis model of human oral carcinoma in nude mice. Journal of The Japanese Stomatological Society 1993; 42: 534-540.

[10] MYERS JN, HOLSINGER FC, JASSER SA, BEKELE BN, FIDLER IJ. An orthotopic nude mouse model of oral tongue squamous cell carcinoma. Clin Cancer Res 2002; 8: 293-298.

[11] PATEL V, MARSH CA, DORSAM RT, MIKELIS CM, MASEDUNSKAS A, et al. Decreased lymphangiogenesis and lymph node metastasis by mTOR inhibition in head and neck cancer. Cancer Res 2011; 71: 7103-7112.

[12] UMEDA M, YOKOO S, KOMORI T, NISHIMATSU N, SHIBUYA Y, et al. Experimental model of invasion and metastasis by orthotopic transplantation of oral squamous and adenoid cystic carcinomas into the tongue of nude mice. Br J Oral Maxillofac Surg 2001; 39: 276-380.

[13] SUN R, ZHANG JG, GUO CB. Establishment of cervical lymph node metastasis model of squamous cell carcinoma in the oral cavity in mice. Chin Med J (Engl) 2008; 121: 1891-1895.

[14] MATSUI T, OTA T, UEDA Y, TANINO M, ODASHIMA S. Isolation of a highly metastatic cell line to lymph node in human oral squamous cell carcinoma by orthotopic implantation in nude mice. Oral Oncol 1998; 34: 253-256. 